\title{
Primary sarcomatoid carcinoma of the jejunum with massive intra-abdominal hemorrhage: A case report and review of the literature
}

\author{
BIN ZHANG $^{1}$, BO CHENG $^{2}$, LINXIONG WANG $^{3}$, KE ZHAO $^{1}$, GUANG-ZUAN ZHUO $^{1}$ and JIAN-HUA DING ${ }^{1}$
}

Departments of ${ }^{1}$ Colorectal Surgery and ${ }^{2}$ Clinical Pathology, The General Hospital of the PLA Rocket Force, Beijing 100088;

${ }^{3}$ Cancer Center Lab, Chinese People's Liberation Army General Hospital \& Beijing Key Laboratory

of Cell Engineering \& Antibody, Beijing 100853, P.R. China

Received October 26, 2015; Accepted February 25, 2016

DOI: $10.3892 / \mathrm{mco} .2016 .809$

\begin{abstract}
Primary sarcomatoid carcinoma of the jejunum is an extremely rare condition, with only 16 cases reported in the literature to date. We herein report an additional case of a giant sarcomatoid carcinoma of the jejunum in a 62-year-old male patient, presenting as massive intra-abdominal hemorrhage. During emergency laparotomy, $\sim 5$ litres of bloody ascites was found in the peritoneal cavity and the tumor was located in the proximal jejunum. The tumor involved the entire wall of the jejunum and had directly invaded the neighboring parietal peritoneum, omentum, transverse colon and mesentery, with metastatic lymph nodes. The patient underwent palliative resection of the tumor; however, the course was rapidly progressive and he succumbed to progression of abdominal and liver metastases 4 weeks after surgery. The tumor was found to be positive for epithelial and mesenchymal markers on immunohistochemical analysis. This case emphasizes the aggressive clinical course and metastatic nature of this malignant tumor, with a supplementary review of the previously published literature.
\end{abstract}

\section{Introduction}

Primary sarcomatoid carcinoma (SCA) is an extremely rare biphasic malignant tumor, composed of malignant epithelial and mesenchymal cells (1-3). A variety of terms, including carcinosarcoma, pleomorphic carcinoma and anaplastic giant-cell carcinoma have been previously used to describe this tumor; however, based on its ultrastructural and immunohistochemical characteristics, SCA is currently the most universally accepted term and is recommended for routine

Correspondence to: Dr Jian-Hua Ding, Department of Colorectal Surgery, The General Hospital of the PLA Rocket Force, 16 Xinjiekouwai Road, Xicheng, Beijing 100088, P.R. China E-mail: thalarus@126.com

Key words: sarcomatoid carcinoma, jejunal tumor, small intestine, programmed death-ligand 1, Ki-67 use in diagnostic surgical reports $(2,3)$. Although these tumors have been described in various locations throughout the digestive system (1), to the best of our knowledge, only 16 cases of primary SCA of the jejunum have been reported in the literature to date. Notably, SCA is known to be more aggressive compared with other small intestinal tumors (4), and patients with this disease have a significantly worse prognosis due to the tumor's metastatic nature and aggressive clinical course. We herein report an additional case of jejunal SCA with massive intra-abdominal hemorrhage occurring in a 62-year-old male patient, followed by a review of the relevant literature. Written informed consent was obtained from the patient's family for the publication of the details of this case.

\section{Case report}

A 62-year-old man was admitted with complaints of intermittent abdominal pain and distension for 2 years. Two weeks prior to hospitalization, the patient suffered from continuous abdominal distension and he developed new symptoms including melaena, dizziness and fatigue. There had been no recent change in bowel habits. The patient was diagnosed with severe anaemia and hypoproteinemia at a local hospital, but his symptoms were not alleviated following conservative treatment and blood transfusion. An abdominal computed tomography (CT) scan revealed a bulky mass, sized $11 \times 6.5 \times 4 \mathrm{~cm}^{3}$, possibly originating from the small intestine (Fig. 1A). The patient was then admitted to our hospital for further investigation and treatment.

On physical examination, the patient had a fever of $38^{\circ} \mathrm{C}$ and appeared pale, with a pulse rate of 120 beats/min and a blood pressure of 100/60 $\mathrm{mmHg}$. There was abdominal distension and mild right upper quadrant tenderness to palpation. Shifting dullness and high-pitched tinkling bowel sounds were negative. The patient had flatus and passed a small amount of stool daily, but the fecal occult blood test was positive, indicating unremitting gastrointestinal bleeding. The blood test results for hemoglobin ( $\mathrm{Hb})$, hematocrit (Hct), red blood cell (RBC) count and albumin (Alb) were $7.1 \mathrm{~g} / \mathrm{dl}, 21.8 \%$, $2.42 \times 10^{12} / 1$ and $31.2 \mathrm{~g} / 1$, respectively. Abdominal radiography revealed intestinal gas accumulation. The carcinoembryonic 


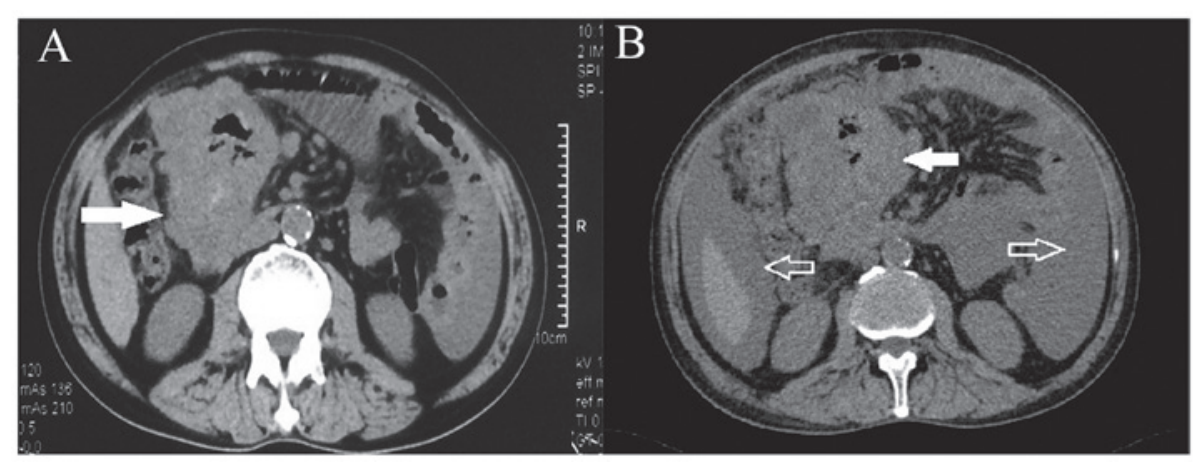

Figure 1. Abdominal computed tomography images in a 62-year-old man with intermittent abdominal pain and distension. (A) Prior to hospitalization, axial image showing an irregular mass (solid arrow) arising from the small bowel. (B) In addition to the primary tumor (solid arrow), massive ascites (open arrows) was identified prior to emergency laparotomy.

antigen and carbohydrate antigen 19-9 levels were within the normal range; other tumor markers were not measured. Although the patient's white blood cell count was elevated to $10.76 \times 10^{9} / 1$ (78.5\% neutrophils), the hemoculture, urine analysis, radiography and physical examination revealed no conspicuous cause of the fever.

Following medication therapy and blood transfusion for $24 \mathrm{~h}$, the patient complained of progressive aggravation of the abdominal distension, palpitations and shortness of breath on effort. Notably, the blood test results for $\mathrm{Hb}$ $(4.9 \mathrm{~g} / \mathrm{dl})$, Hct $(16 \%)$ and RBC count $\left(1.72 \times 10^{12} / 1\right)$ were prominently decreased. In addition to the primary tumor, massive ascites was also observed on CT re-examination (Fig. 1B). Additionally, abdominal paracentesis revealed the presence of free blood in the peritoneal cavity. The patient underwent emergency explorative laparotomy, as there was a high possibility of cancerous and gastrointestinal bleeding, as well as other complications.

During the operation, $\sim 5$ litres of bloody ascites was found in the peritoneal cavity and a $12 \times 10 \mathrm{~cm}^{2}$-mass with necrosis and hemorrhage was identified in the jejunum $(50 \mathrm{~cm}$ from the ligament of Treitz). The mass involved the entire wall of the jejunum and directly invaded the neighboring parietal peritoneum, omentum, transverse colon and mesentery, with regional lymph node enlargement (Fig. 2). However, there was no palpable mass on the surface of liver or other organs in the abdominal cavity. The advanced stage of the tumor did not allow for curative resection; therefore, a portion of the jejunum with the attached mesentery and segmental transverse colon were palliatively removed. A total of 7 mesenteric lymph nodes were also resected and metastasis was confirmed in one lymph node (TNM stage pT4N1M0).

Microscopically, the tumor exhibited prominently malignant characteristics. The scattered and diffuse distribution of tumor cells was growing in a poorly cohesive sarcomatoid formation, supported by scanty fibrous tissue. Polygonal and round tumor cells were significantly atypical, with abundant eosinophilic cytoplasm and pleomorphic giant nuclei (Fig. 3A). The mitotic figures averaged 25-30 per 10 high-power fields. Prominent vascular invasion was also noted (Fig. 3B). Immunohistochemical staining revealed a diffuse and strongly positive reaction for vimentin (Fig. 3C) and markedly positive staining for Pan-cytokeratin (CK) (Fig. 3D), CK7, CK18 (Fig. 3E), CK19, epithelial membrane antigen (Fig. 3F), Ki-67 (Fig. 3G) and programmed deathligand 1 (PD-L1) (Fig. 3H). By contrast, CD34, CD117, discovered on GIST-1, human melanoma black-45, melan-A, S-100, smooth muscle actin, desmin, myogenic differentiation 1, myogenin and myoglobin all appeared to be negative. The pathological test results were indicative of SCA of the jejunum.

The patient postoperatively developed bloody ascites (540-1,950 ml/day), in which malignant cells were detected. The tumor exhibited an aggressive clinical course and the patient succumbed to rapid progression of the abdominal and liver metastases 4 weeks after surgery. No autopsy was performed.

\section{Discussion}

In contrast to the stable or declining trends for most cancers, the incidence rate for cancers of the small intestine increased from 2007 through to 2011 (5). Adenocarcinoma, neuroendocrine tumors, sarcomas and lymphomas are the four most common malignant tumors arising in the small intestine (6). Primary SCA of the small bowel is rare and it primarily occurs in the ileum, followed by the jejunum and duodenum (2). Only 16 cases of jejunal SCA have been reported in the literature to date (Table I). These tumors normally affect middle-aged to elderly patients with a mean age at presentation of 59.1 years (range, 35-85 years). Reid-Nicholson et al reported that SCA of the small intestine is more prevalent in male patients, with a male:female ratio of 1.5:1.0 (2); however, our data revealed no significant difference in patients with jejunal SCA, with a male:female ratio of 0.89:1.0. Most SCAs of the jejunum are typically single tumors, but 2 patients exhibited multifocal primary tumors (7).

In a series of 14 cases with complete clinical and pathological data (Table II), anemia was the most common symptom, followed by abdominal pain, palpable abdominal mass, gastrointestinal bleeding, weight loss and fatigue. Two patients (including the present case) presented with cancerous bleeding that required emergency laparotomy (9), and another 2 patients experienced tumor perforation with subphrenic free air and acute peritonitis $(11,13)$. Additionally, shortness of breath on effort, palpitations, vomiting, fever, nausea, diarrhea, dyspepsia and anorexia may also be the initial symptoms of patients with primary SCA of the jejunum. Therefore, the clinical manifes- 


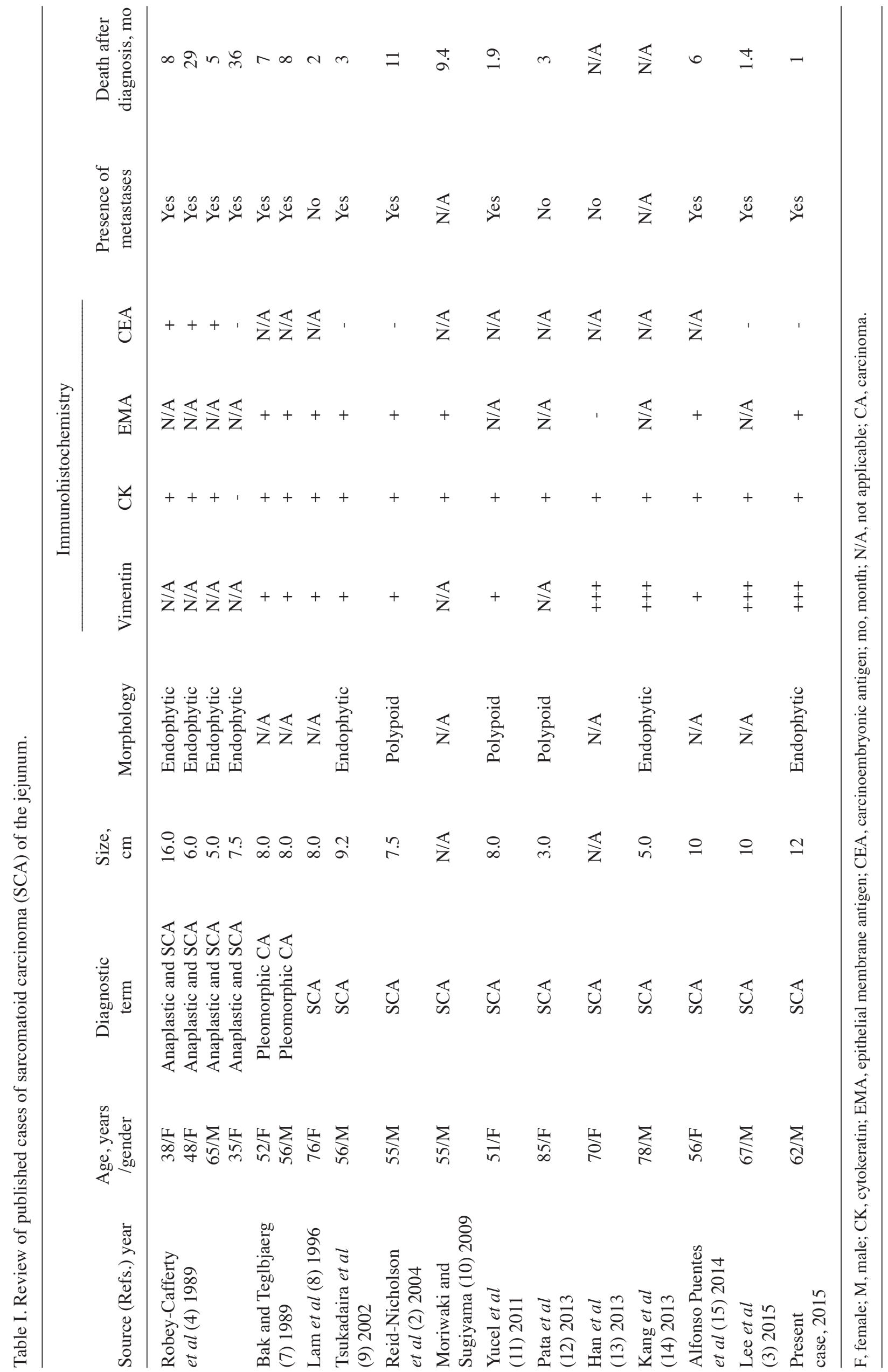


Table II. Summary of clinicopathological characteristics of patients with sarcomatoid carcinoma (SCA) of the jejunum.

\begin{tabular}{|c|c|}
\hline Characteristics & $\begin{array}{c}\text { No. of } \\
\text { patients }(\%)\end{array}$ \\
\hline \multicolumn{2}{|l|}{ Clinical presentation $(n=14)$} \\
\hline Anemia & $8(57.1)$ \\
\hline Abdominal pain & $6(42.9)$ \\
\hline Abdominal mass & $4(28.6)$ \\
\hline Gastrointestinal bleeding & $4(28.6)$ \\
\hline Weight loss & $4(28.6)$ \\
\hline Fatigue & $4(28.6)$ \\
\hline Small intestinal obstruction & $2(14.3)$ \\
\hline Shortness of breath on effort & $2(14.3)$ \\
\hline Palpitations & $2(14.3)$ \\
\hline Subphrenic free air & $2(14.3)$ \\
\hline Vomiting & $2(14.3)$ \\
\hline Fever & $2(14.3)$ \\
\hline Nausea & $1(7.1)$ \\
\hline Diarrhea & $1(7.1)$ \\
\hline Dyspepsia & $1(7.1)$ \\
\hline Anorexia & $1(7.1)$ \\
\hline \multicolumn{2}{|c|}{ Greatest dimension of tumor, $\mathrm{cm}(\mathrm{n}=12)$} \\
\hline$<5$ & $1(8.3)$ \\
\hline $5-10$ & $9(75.0)$ \\
\hline$>10$ & $2(16.7)$ \\
\hline \multicolumn{2}{|l|}{ Depth of invasion $(n=12)$} \\
\hline Muscularis propria & $1(8.3)$ \\
\hline Subserosal layer & $2(16.7)$ \\
\hline Full thickness of the wall & $9(75.0)$ \\
\hline \multicolumn{2}{|l|}{ Regional lymph nodes $(n=10)$} \\
\hline Metastasis & $9(90.0)$ \\
\hline No metastasis & $1(10.0)$ \\
\hline \multicolumn{2}{|l|}{ Distant metastasis $(\mathrm{n}=12)$} \\
\hline Lung & $6(50.0)$ \\
\hline Liver & $3(25.0)$ \\
\hline Para-aortic lymph nodes & $2(16.7)$ \\
\hline Supraclavicular lymph nodes & $2(16.7)$ \\
\hline Cervical lymph nodes & $1(8.3)$ \\
\hline Axillary lymph nodes & $1(8.3)$ \\
\hline Pelvic bone & $1(8.3)$ \\
\hline Brain & $1(8.3)$ \\
\hline \multicolumn{2}{|c|}{ Lymphovascular or perineural invasion $(n=11)$} \\
\hline Yes & $6(54.5)$ \\
\hline No & $5(45.5)$ \\
\hline \multicolumn{2}{|c|}{ Extension into adjacent organs $(n=6)$} \\
\hline Small bowel loops & $3(50.0)$ \\
\hline Colon & $2(33.3)$ \\
\hline Mesentery & $2(33.3)$ \\
\hline Omentun & $2(33.3)$ \\
\hline Ovary & $1(16.7)$ \\
\hline
\end{tabular}

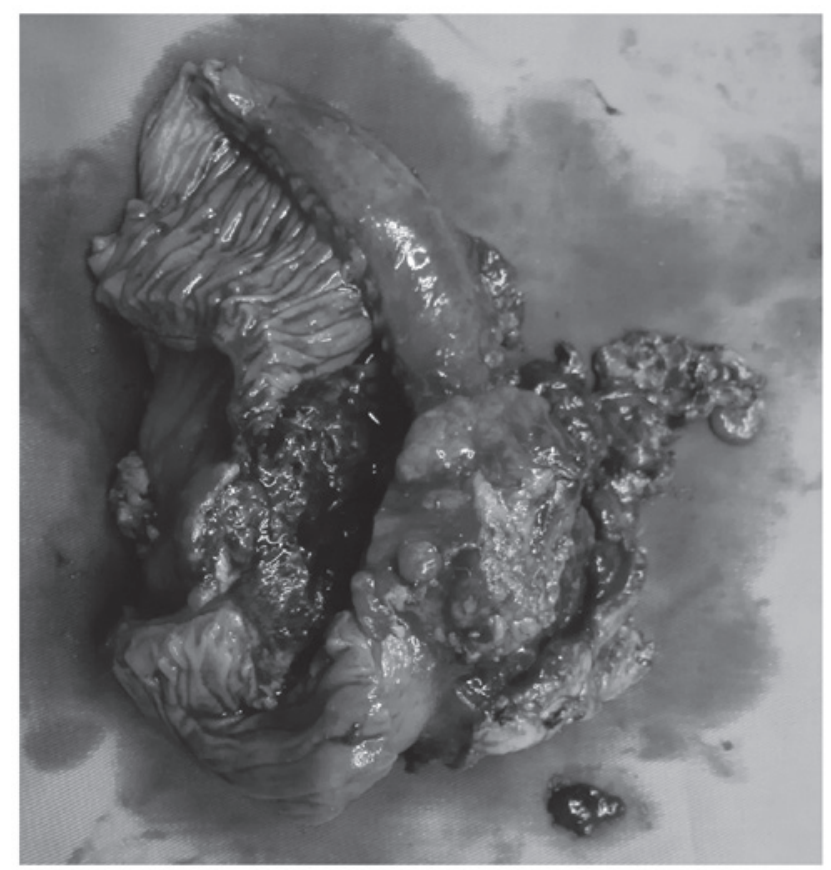

Figure 2. Macroscopic resected specimen exhibiting an endophytic mass with central ulceration and necrosis in the jejunum.

tations of jejunal SCA are remarkably non-specific, which is the primary cause that the majority of such tumors are already advanced at the time of diagnosis. However, it should be noted that the presence of severe anemia and/or abdominal pain is highly suggestive of this rare type of small intestinal malignancies.

Adenocarcinomas of the small bowel tend to be smaller at the time of clinical presentation (4). On the contrary, SCAs are typically endophytic or polypoid masses with central ulceration, averaging $8.21 \mathrm{~cm}$ in the greatest dimension (range, 3-16 cm), and are frequently necrotic and hemorrhagic. As shown in Table II, the majority of primary tumors involved the entire wall of the jejunum with regional lymph node metastasis, and directly invaded neighboring structures, including small bowel loops, colon, mesentery, omentum and ovaries. Moreover, prominent lymphovascular or perineural invasion was found in $54.5 \%$ of the patients. Although all the patients with primary SCAs underwent surgical resection, these tumors progress and metastasize rapidly. The most common metastatic locations are the lung, distant lymph nodes and liver, while the brain and pelvic bones may also be involved. Of the 17 cases reported (including the present case), $100 \%$ of the patients with complete follow-up succumbed to the disease, and $80 \%$ had metastatic or recurrent disease at death, indicating the aggressive and metastatic nature of this tumor. Overall, patients with jejunal SCA have an extremely poor prognosis and the average survival time after diagnosis is only 8.8 months (range, 1-36 months).

Histologically, SCA may exhibit a monophasic or biphasic pattern (4). A predominance of the mesenchymal-like component, with minimal to absent epithelioid areas, characterizes the typical monophasic pattern, whereas biphasic tumors display a mixture of epithelial-like and mesenchymal-like cells, as in the present case. As SCA cannot be definitively diagnosed 


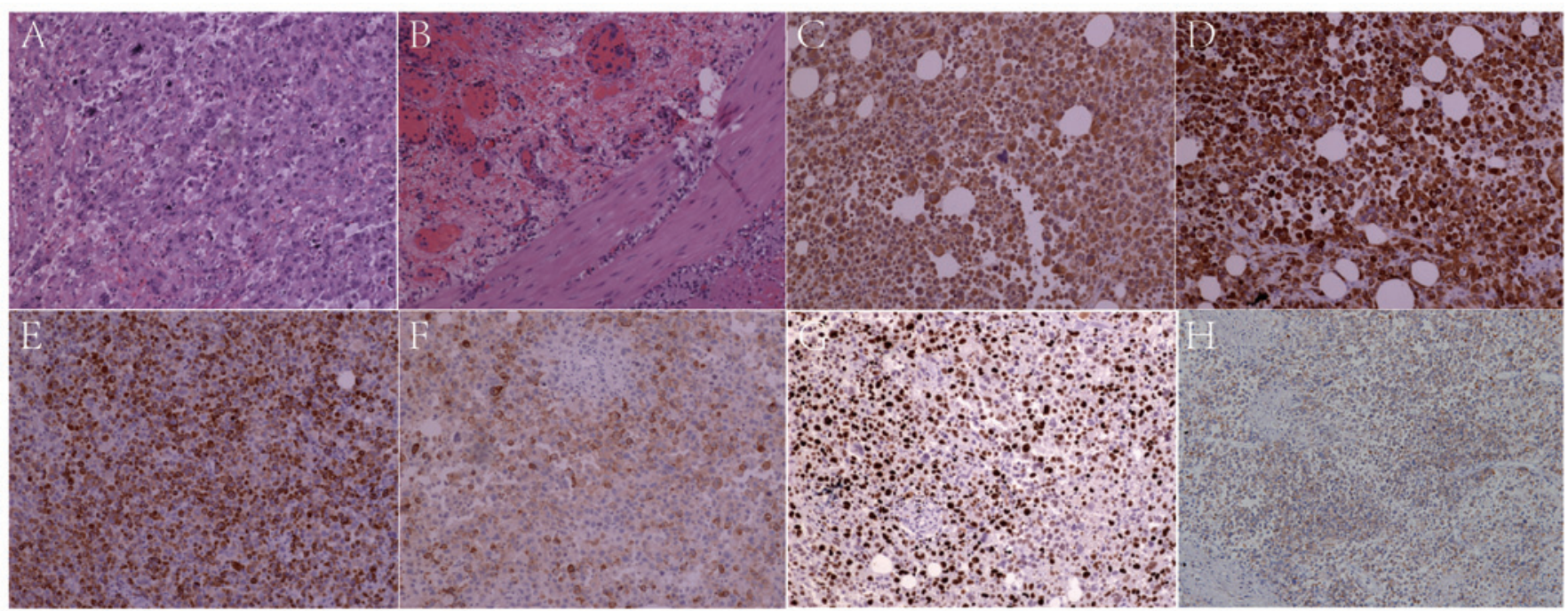

Figure 3. Pathological findings of the tumor. (A) Polygonal-shaped tumor cells exhibiting high atypia and increased mitotic activity. (B) Prominent vascular invasion was also noted (hematoxylin and eosin staining, magnification x100). The tumor cells displayed (C) diffuse strong staining for vimentin, and were strongly positive for (D) Pan-CK, (E) CK18, (F) EMA, (G) Ki-67 and (H) PD-L1 on immunohistochemistry. CK, cytokeratin; EMA, epithelial membrane antigen; PD-L1, programmed death-ligand 1.

based on hematoxylin and eosin staining alone, a wide panel of immunohistochemical biomarkers is crucial for differential diagnosis (1-3). Our immmunohistochemical staining results revealed markedly positive expression of vimentin and Pan-CK, indicating that the sarcomatous component displayed epithelial and mesenchymal differentiation; hence, the diagnosis of SCA was confirmed. Intriguingly, Han et al reported that their patient exhibited a relatively favorable prognosis, which may be attributed to the low positivity for Ki-67 in the tumor (13). However, the positivity for Ki-67 was found to be $>75 \%$ in the present case, which was associated with an invasive and aggressive clinical course. Therefore, whether Ki-67 may be used as a potential prognostic marker for patients with jejunal SCA remains to be further elucidated.

Compared with conventional small intestinal tumors, SCA is significantly more aggressive and traditional therapeutic methods are generally ineffective. Recently, clinical trials using monoclonal antibodies targeting immune checkpoint programmed death protein 1 (PD-1) and its ligand, PD-L1, reported promising antitumor activity in several malignancies. Upregulated PD-L1 expression on tumor cells is considered to mediate immune evasion via activation of the PD-1/PD-L1 pathway and suppression of effector immune responses (16). Interestingly, we observed a distinct expression of the PD-L1 protein in primary SCA of the jejunum, which is consistent with a previous report showing PD-L1 upregulation in sarcomatoid lung carcinomas (17), indicating the potential of PD-L1-targeted immunotherapy for the treatment of this type of cancer.

In conclusion, patients with primary SCA of the jejunum exhibit a significantly worse prognosis due to the metastatic nature and aggressive clinical course of this tumor. Early detection and treatment through thorough examination and radical resection may improve patient prognosis. The present case also suggests the potential value of PD-L1 and Ki-67 as an important clinical or prognostic biomarker in jejunal SCA.
However, their precise role in tumor pathogenesis requires further investigation.

\section{Acknowledgements}

This study was supported by a grant from the Clinical Research Fund of Beijing Municipal Science \& Technology Commission (no. Z151100004015013).

\section{References}

1. Fukuda T, Kamishima T, Ohnishi Y and Suzuki T: Sarcomatoid carcinoma of the small intestine: Histologic, immunohistochemical and ultrastructural features of three cases and its differential diagnosis. Pathol Int 46: 682-688, 1996.

2. Reid-Nicholson M, Idrees M, Perino G and Hytiroglou P Sarcomatoid carcinoma of the small intestine: A case report and review of the literature. Arch Pathol Lab Med 128: 918-921, 2004.

3. Lee HM, Cho MS and Kim YI: A surgically resected large sarcomatoid carcinoma of the jejunum: A case report and literature review. J Gastric Cancer 15: 143-146, 2015.

4. Robey-Cafferty SS, Silva EG and Cleary KR: Anaplastic and sarcomatoid carcinoma of the small intestine: A clinicopathologic study. Hum Pathol 20: 858-863, 1989.

5. Siegel RL, Miller KD and Jemal A: Cancer statistics, 2015. CA Cancer J Clin 65: 5-29, 2015.

6. Reynolds I, Healy P and Mcnamara DA: Malignant tumours of the small intestine. Surgeon 12: 263-270, 2014.

7. Bak M and Teglbjaerg PS: Pleomorphic (giant cell) carcinoma of the intestine: An immunohistochemical and electron microscopic study. Cancer 64: 2557-2564, 1989.

8. Lam KY, Leung CY and Ho JW: Sarcomatoid carcinoma of the small intestine. Aust N Z J Surg 66: 636-639, 1996.

9. Tsukadaira A, Koizumi T, Okubo Y, Takashi S, Koide N, Arai K, Ota $\mathrm{H}$ and Kubo K: Small-intestinal sarcomatoid carcinoma with superior vena cava syndrome. J Gastroenterol 37: 471-475, 2002.

10. Moriwaki Y and Sugiyama M: Severe anemia inducing preshock caused by sarcomatoid carcinoma of the small intestine. Int Surg 94: 164-170, 2009.

11. Yucel AF, Kocakusak A, Arikan S, Demirbag N, Tarlaci A and Batur S: A rare cause of acute abdomen: Perforated primary sarcomatoid carcinoma of the small intestine - report of a case, with a brief review of the literature. J Cancer Res Ther 7: 348-350, 2011 
12. Pata F, Sengodan M, Tang CB, Kadirkamanathan SS, Harvey M, Zaitoun A, Petkar M and Rotundo A: Concomitant jejunal sarcomatoid carcinoma and gastric GIST in patient with polymyalgia rheumatica: A case report. Int J Surg Case Rep 4: 449-452, 2013.

13. Han N, Han QH, Liu YZ, Li ZC and Li J: Perforated sarcomatoid carcinoma of the jejunum: Case report. Oncol Lett 6: 562-564, 2013.

14. Kang KA, Yoon JH and Kang G: Sarcomatoid carcinoma of the small intestine: A case report and review of the literature. J Korean Soc Radiol 69: 295-299, 2013.
15. Alfonso Puentes N, Jimenez-Alfaro Larrazabal C and García Higuera MI: Sarcomatoid carcinoma of the jejunum presenting as obscure gastrointestinal bleeding in a patient with a history of gliosarcoma. Gastroenterol Rep (Oxf) 2: 150-153, 2014.

16. Brahmer JR, Tykodi SS, Chow LQ, Hwu WJ, Topalian SL, Hwu P, Drake CG, Camacho LH, Kauh J, Odunsi K, et al: Safety and activity of anti-PD-L1 antibody in patients with advanced cancer. N Engl J Med 366: 2455-2465, 2012.

17. Velcheti V, Rimm DL and Schalper KA: Sarcomatoid lung carcinomas show high levels of programmed death ligand-1 (PD-L1). J Thorac Oncol 8: 803-805, 2013. 